\title{
Nervennahrung oder nervende Nahrung - Ernährung in der Neurorehabilitation
}

Barbara Schwencker

Wir brauchen es, wir lieben es, wir tun es: essen. Das Selbstverständlichste der Welt kann aber plötzlich zu einem Problem werden, wenn sich die Frage stellt: Kann ich überhaupt noch richtig essen? Wie viel und was brauche ich wirklich? Und wie kann dieser Bedarf sichergestellt werden? All diese Fragen tauchen täglich bei unseren Patienten in der Neuroreha auf, und nur gemeinsam kann es Pflegekräften, Therapeuten und Ärzten gelingen, jeden einzelnen Patienten optimal zu versorgen.

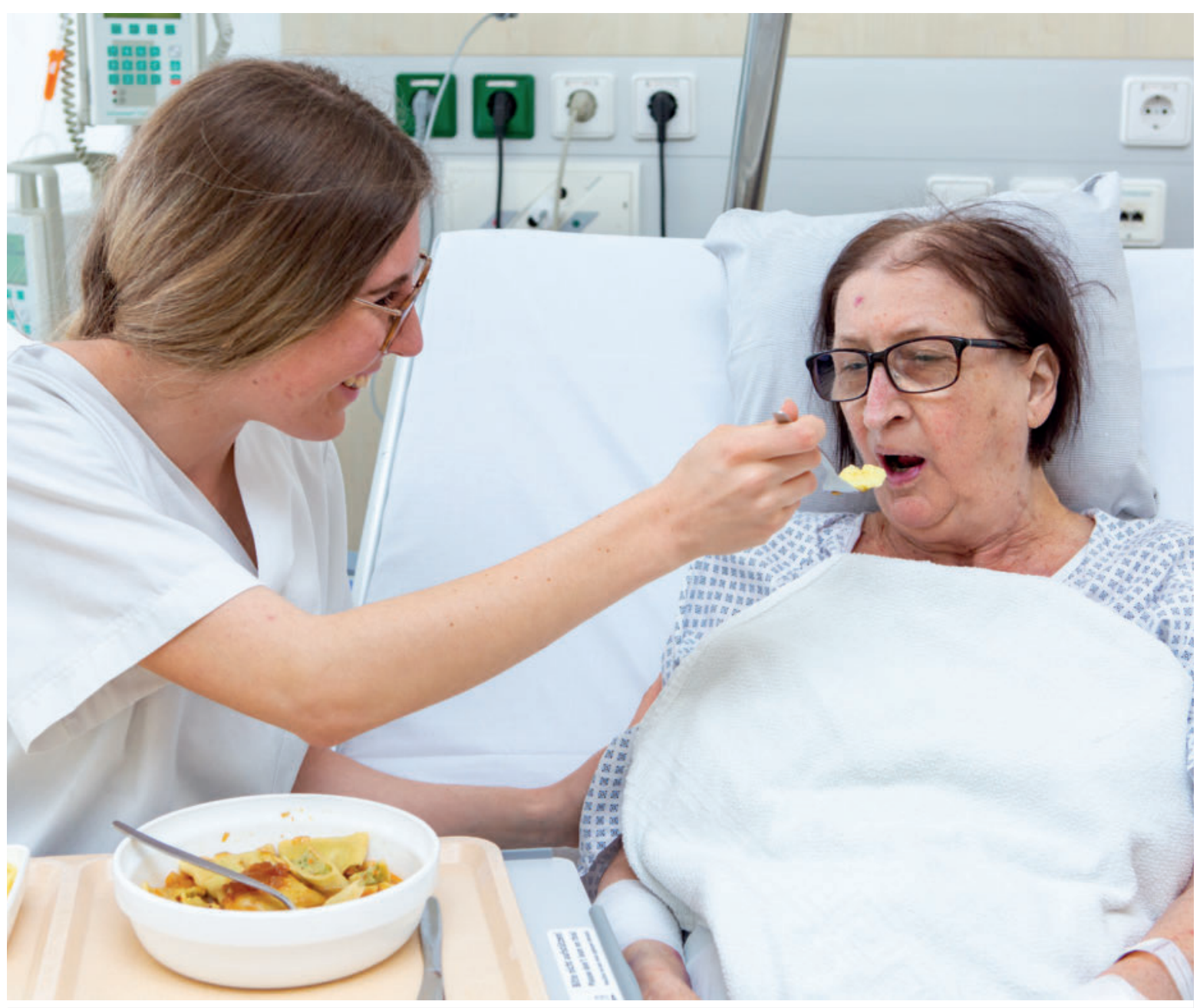

Generell ist zu beachten, dass Frühreha-Patienten deutlich langsamer essen. (Quelle: K. Oborny/Thieme Gruppe; Symbolbild) 
Das Thema Ernährung ist in den letzten Jahren immer präsenter geworden. Man hat erkannt, dass etwas, was eigentlich selbstverständlich ist, bei Patienten einen durchaus relevanten Einfluss auf den Krankheits- bzw. Heilungsverlauf nehmen kann. So gibt es von den unterschiedlichsten Fachgesellschaften jeweils eigene Leitlinien zur Ernährungstherapie sowie nationale und internationale Richtlinien zu diesem Thema. Im Folgenden stütze ich mich auf den LEKuP, den Leitfaden Ernährungstherapie in Klinik und Praxis 2019, die ESPEN Guideline der European Society of Clinical Nutrition and Metabolism sowie den Konsens der GLIM (Global Leadership Initiative on Malnutrition). Die Leitlinie der Deutschen Gesellschaft für Neurologie stammt von 2013 und wird aktuell überarbeitet.

\section{Wie viele Kalorien brauchen unsere Patienten?}

Patienten, die in die neurologische Reha kommen, haben sehr unterschiedliche Vorgeschichten. Man kann zwischen Patienten mit einer neurologischen Grunderkrankung wie z. B. einem Morbus Parkinson, Patienten mit primär internistischen Grunderkrankungen wie Diabetes und Bluthochdruck, die dann einen Schlaganfall erlitten haben, und Patienten, die eigentlich ohne Vorerkrankungen, beispielsweise nach einem Unfall, kommen, unterscheiden. Während Letztgenannte meist erst einen kurzen Krankheitsverlauf hinter sich haben, haben die anderen Patientengruppen häufig schon eine lange Krankheitsgeschichte und damit andere Bedürfnisse hinsichtlich der Ernährung.

Mangelernährung Zu Beginn jeder Behandlung sollte bei allen Patienten ein Screening auf Mangelernährung durchgeführt werden [1]. Mangelernährung kann auch bei Normal- und Übergewichtigen vorliegen und ist durch einen Mangel an Mikronährstoffen und Eiweiß gekennzeichnet. Hierzu stehen verschiedene Tools zur Verfügung: Ein einfacher Screeningtest ist z. B. der Nutritional Risk Score (NRS) 2002 [2]. Dabei gehen verschiedene Faktoren wie Schwere und Dauer der Erkrankung sowie Gewichtsverlust und unzureichende Nahrungsaufnahme ggf. auch schon vor der stationären Behandlung ein. Dieser Test liefert erste Erkenntnisse darüber, ob der Patient für eine Mangelernährung gefährdet ist.

Body Mass Index Des Weiteren müssen das Gewicht und der BMI (Body Mass Index) des Patienten ermittelt werden. Der BMI wird aus Körpergewicht geteilt durch Körpergröße in Meter zum Quadrat errechnet. Im Anschluss daran kann eine entsprechende bedarfsadaptierte Ernährung angeordnet werden. Dies sollte durch den Stationsarzt als ärztliche Anordnung erfolgen. Beim normalgewichtigem Erwachsenen (Body Mass Index 18,5-24,9 nach WHO) ist das in der Regel eine ausgewogene Vollkost, bei der die benötigten Kalorien zu 50-55\% durch Kohlenhydrate, zu 30-35\% durch Fett und durch 0,8g Eiweiß/kg Körpergewicht (KG) und Tag zugeführt werden [3].
Physical Activity Level Bei der Berechnung der benötigten Kalorienmenge kommt neben der Körpergröße und dem Gewicht, die den Ruheverbrauch bestimmen, auch das Physical Activity Level (PAL) zum Tragen. Dabei wird der Grundumsatz (ca. 25-30 kcal/kg KG) mit einem Faktor multipliziert, um den Gesamtbedarf zu ermitteln. So wird der PAL bei bettlägerigen Patienten mit 1,2 und bei mobilen Reha-Patienten mit 1,4 veranschlagt. Bei körperlich schwer arbeitenden Menschen, aber auch bei bestimmten Erkrankungen kann der PAL bis 2 steigen. So ist sowohl bei Patienten mit Morbus Parkinson als auch mit Chorea Huntington der Energiebedarf deutlich gesteigert. Bei geriatrischen Patienten (> 70 Jahre) wird ein niedrigerer Grundumsatz angenommen ( $20 \mathrm{kcal} / \mathrm{kg} \mathrm{KG)}$.

Nahrungsergänzung und Diät In Hinblick auf die mancherorts vielgepriesenen Nahrungsergänzungsmittel gibt es für neurologische Patienten keine Vorteile. Ebenso gibt es keine spezielle Diät, die den Krankheitsverlauf wissenschaftlich bewiesen verbessert [1]. Im Vordergrund steht das Vermeiden von Mängeln - sowohl bei Kalorien als auch bei Eiweiß, Vitaminen und Spurenelementen. Bei einem laborchemisch nachgewiesenen Mangel sollte dann eine Substitution erfolgen. Eine Einnahme spezieller Vitaminpräparate über den normalen Bedarf hinaus ist nicht sinnvoll. In Einzelfällen kann der Bedarf allerdings erhöht sein, beispielsweise der Eiweißbedarf bei Patienten mit Dekubitus. Dann kann unter Berücksichtigung aller Nebenwirkungen (in diesem Fall Belastung für die Niere bei Niereninsuffizienz) eine zusätzliche Eiweißsubstitution nötig werden. Diese speziellen Kostformen sollten dann mit einem Internisten bzw. einem Arzt für Ernährungsmedizin abgesprochen werden.

\section{Wie erkennt man eine ausreichende Versorgung?}

Zunächst spielt die genaue Dokumentation der Nahrungszufuhr eine große Rolle. So haben wir es in der Neurologie mit einer sehr unterschiedlichen Patientenklientel zu tun. Da gibt es den Patienten mit tiefem Querschnitt, der komplett selbstständig essen kann, ebenso wie den an Parkinson erkrankten Patienten, der unter Umständen eine Schluckstörung hat, neben dem Patienten mit schwerem Apoplex, der überhaupt nicht oral ernährt werden kann.

Enterale Ernährung Bei Patienten, die über eine nasogastrale Sonde oder eine perkutane Gastroenterostomie (PEG) ernährt werden, ist die Nahrungszufuhr klar aus der Verordnungsübersicht zu erkennen. Eine zusätzliche Dokumentation ist dann im Allgemeinen nicht nötig. Sollte es aber aus irgendwelchen Gründen nicht möglich sein, die verordneten Nahrungsmengen zu applizieren, muss unbedingt der Arzt informiert werden. So kann beispielsweise anhaltender Reflux ebenso wie Erbrechen ein ernst zu nehmendes Problem sein. 
Orale Ernährung Bei Patienten, die orale Kost zu sich nehmen, hat sich das sogenannte Tellerdiagramm bewährt. Dabei wird nach Ende der Mahlzeit dokumentiert, wie viele Viertel des Tellerinhalts gegessen wurden - 1/4 bis $4 / 4$. Anschließend kann die Kalorienzahl relativ einfach berechnet werden: Wird ein Mittagessen mit $800 \mathrm{kcal}$ berechnet und es wurde der Teller nur halb leer gegessen, entspricht das 400 kcal. Standardmäßig haben Frühstück und Abendessen $600 \mathrm{kcal}$, das Mittagessen wird mit 800 kcal gezählt. Unter Umständen kommen dann noch Trinknahrung oder Getränke dazu. Liegt die Verzehrmenge regelmäßig unter $3 / 4$ des Angebots, müssen Maßnahmen ergriffen werden.

Wichtig ist, dass sowohl Therapeuten als auch Pflegende mit diesem System vertraut sind, denn jeder, der den Patienten beim Essen betreut, sollte dies gewissenhaft dokumentieren. Ebenso sollten Getränke beispielsweise im Rahmen der Therapie oder zwischen den Therapien gut dokumentiert werden. Nur so kann man abschätzen, ob der Patient ausreichend mit Nahrung und Flüssigkeit versorgt ist.

Gewichtskontrolle Neben verschiedenen Laborparametern ist die regelmäßige Gewichtskontrolle eine wichtige Maßnahme zur Beurteilung einer ausreichenden Nährstoffversorgung, aber leider nicht immer die zuverlässigste. Schnelle und ausgeprägte Gewichtsschwankungen können durch Wassereinlagerungen bedingt sein. Ebenfalls kann man nicht differenzieren, ob es sich bei dem Gewicht um Fett oder Muskelmasse handelt. So kann das Körpergewicht gleich bleiben, wenn der Patient an Muskelmasse verliert und Fett einlagert - ein Effekt, der unerwünscht ist und sich bei der Mobilisation negativ niederschlagen kann.

Es gibt verschiedene Möglichkeiten, das Körperfett zu messen, beispielsweise die BIA-Messung (bioelektrische Impedanzanalyse); allerdings sind diese Messungen mit einem zusätzlichen apparativen und zeitlichen Aufwand verbunden. Weitere Möglichkeiten sind Testungen aus der Geriatrie, die den Schwerpunkt auf Sarkopenie und Frailty legen und einen Hinweis auf Eiweißmangel und Muskelverlust geben (z. B. Sarc-F-Fragebogen, Knochendichtemessung).

\section{Ist es sinnvoll, übergewichtige Patienten in der Reha auf Normalgewicht zu bringen?}

Wenn man sich unsere Patienten unter den oben genannten Gesichtspunkten ansieht, wird schnell klar, dass auch übergewichtige Personen häufig mangelernährt sind und zu wenig Muskulatur aufweisen. Es hat dann keinen Sinn, diese Patienten auf eine strenge Diät zu setzen und auf bessere Mobilisation zu hoffen. Der Körper greift in Hun- gerzuständen zuerst die Muskulatur an, erst in zweiter Linie werden die Fettreserven mobilisiert. Und dieser Vorgang macht sich im Alter immer stärker bemerkbar. Die Deutsche Gesellschaft für Ernährungsmedizin e. V. (DGEM) rät daher explizit von Nahrungsrestriktion bei älteren Patienten ab [4]. Nur in ausgesuchten Fällen kann unter engmaschiger Kontrolle eine langsame Gewichtsreduktion angestrebt werden (Kaloriendefizit von $500 \mathrm{kcal} / \mathrm{d}$ ).

\section{Was tun mit essunwilligen Patienten?}

Aber auch Patienten, die eigentlich in der Lage wären, zu essen, kommen manchmal nicht auf die benötigten Kalorienmengen. Wichtig ist es dann, herauszufinden, warum der Patient nicht isst. Eine besonders kritische Phase ist hierbei der Übergang von der enteralen zur oralen Kost. Hierbei können das visuell und geschmacklich oft wenig ansprechende passierte Essen ebenso wie eine depressive Verstimmung, eine Antriebsstörung oder ein Aufmerksamkeitsdefizit ursächlich sein. Appetitlosigkeit und Übelkeit sind zudem durchaus häufige Medikamentennebenwirkungen (z. B. bei SSRI, Levodopa, Digoxin und Metformin). Die Tatsache, dass das Essen „nicht schmeckt“, kann genauso problematisch sein wie eine subjektive oder objektive Unverträglichkeit.

Generell ist zu beachten, dass Frühreha-Patienten deutlich langsamer essen. Bevor der Teller halb voll wieder abgeräumt wird, sollte sichergestellt sein, dass der Patient auch wirklich fertig ist mit dem Essen.

Abklärung organischer Ursachen Wichtig ist es natürlich, organische Ursachen wie beispielsweise eine Schluckstörung auszuschließen. So sollte jeder Patient, der eine Erkrankung hat, die klassischerweise mit Schluckstörungen einhergeht (z. B. Apoplex, Morbus Parkinson, Amyotrophe Lateralsklerose), möglichst bald durch einen Schlucktherapeuten beurteilt werden. Auch ein Passagehindernis oder ein Magengeschwür können Probleme machen, ggf. ist eine weitergehende endoskopische Abklärung mittels Gastroskopie indiziert. Eine Obstipation stört den Appetit empfindlich, ebenso wie ein vielleicht bestehender Schwindel.

Grundsätzlich sollte man immer versuchen, mit dem Patienten zu reden, um das Kernproblem zu erkennen und eine Lösung zu finden. Oft kann man mit kleinen Änderungen im Speiseplan - ein zusätzlicher Brei oder Joghurt - gute Erfolge erzielen. Eine Banane extra wird meist lieber gegessen als die Tablette Kalium.

Trinken Eine ausreichende Trinkmenge ist insbesondere für die kognitiven Funktionen relevant. Durch das verminderte Durstgefühl der oft alten, neurologisch erkrankten Patienten wird die erforderliche Trinkmenge häufig nicht erreicht. So sollten Patienten, die trinken können, aber ein Problem mit der Menge haben, immer wieder von allen 
mit ihnen befassten Personengruppen (Pflege, Therapeuten, Angehörige) dazu animiert werden. Das Umfeld des Patienten muss so gestaltet werden, dass dieser gut und möglichst selbstständig trinken kann (Höhe des Kopfteils, Trinkbecher in Reichweite). Die Aufbilanzierung mittels Infusionen ist als Ultima Ratio zwar möglich, sollte aber die Ausnahme bleiben.

\section{Ernährungsmanagement}

Alle Maßnahmen, die dazu dienen, den Patienten gut zu ernähren, werden im Ernährungsmanagement zusammengefasst. Dies beinhaltet minimal: Berechnung des Energiebedarfs (s. o.), wöchentliche Gewichtskontrollen und tägliche Erfassung der Verzehr- und Trinkmenge.

Ernährungsmanager In der Praxis hat es sich bewährt, auf jeder Station Ernährungsmanager einzusetzen. Dies sind Pflegekräfte, die ihr spezielles Augenmerk auf die Ernährung der Patienten richten, das heißt Kontrolle der Zufuhr und der Gewichtsverläufe. Sollten sich hierbei Auffälligkeiten zeigen, wird der Stationsarzt verständigt. Zudem treffen sich alle Ernährungsmanager vierteljährlich mit dem Arzt für Ernährungsmedizin im Haus, um allgemeine Prozessthemen oder Probleme jeder Art zu besprechen. Themen sind neben fachlichen Fragen (z. B. welche Sondenkostsorten) auch Prozessoptimierungen, z. B. wer die Patienten wiegt und in welchen Abständen. Die enge Zusammenarbeit von Pflege und Ärzten ist hierbei sehr wichtig, weil die ausgefeilteste Ernährungstherapie nichts hilft, wenn die Umsetzung nicht funktioniert.

Gewichtsmessung Bezüglich der Gewichtsmessung empfiehlt es sich, diese in andere Routinetätigkeiten einzubinden, z. B. ein Wiegen bei Aufnahme durch die Diagnostik im Rahmen des Aufnahme-EKGs. Die weiteren Kontrollen finden auf Station statt - durchaus auch im Rahmen der Therapien, beispielsweise als Spaziergang über die Bodenwaage (die Ungenauigkeit der Messung zu unterschiedlichen Tageszeiten ist im Verlauf zu vernachlässigen).

\section{Schlussbetrachtung}

Essen ist ein wichtiger Teil unseres Lebens, es hat viel mit Lebensqualität, aber auch mit Selbstbestimmung und Selbsterhalt zu tun. Je nach Grad ihrer Selbstständigkeit sind unsere Patienten diesbezüglich stark von uns abhängig. Nur durch eine gute Zusammenarbeit von Ärzten, die die Nahrung anordnen, Pflege, die die Nahrung verabreicht, und Therapeuten, die den körperlichen Zustand ihrer Patienten im Blick haben, kann eine optimale Versorgung erreicht werden.

\section{Autorinnen/Autoren}

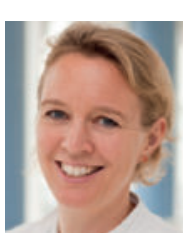

Dr. med. Barbara Schwencker

Fachärztin für Innere Medizin, Ernährungsmedizinerin DAEM/DGEM, Hypertensiologin DHL, zuständig für die internistischen Belange der Patienten sowie für Ernährung und Wundmanagement in der neurologischen Reha der Schön Klinik Bad Aibling

Korrespondenzadresse

Dr. med. Barbara Schwencker

Schön Klinik Bad Aibling SE \& Co. KG

Kolbermoorer Straße 72

83043 Bad Aibling

Deutschland

\section{Literatur}

[1] Schuetz P et al. Individualised nutritional support in medical inpatients at nutritional risk: A randomised clinical trial. Lancet 2019; 393: 2312-2321

[2] Schütz T, Valentini L, Plauth M. Screening auf Mangelernährung nach den ESPEN-Leitlinien 2002. Aktuel Ernaehr Med 2005; 30: 99-103

[3] Hauner $\mathrm{H}$ et al. Leitfaden Ernährungstherapie. Aktuel Ernahrungsmed 2019; 44: 384-419

[4] Kreymann KG, Okenga J. Klug entscheiden ... in der Ernährungsmedizin. Dtsch Ärztebl 2019; 116: A-1956, B-1602, C-1570

[5] Burgos R, Breton I, Cereda E et al. ESPEN guideline clinical nutrition in neurology. Clinical Nutrition 2018; 37: 354-396

Bibliografie

DOI https://doi.org/10.1055/a-1284-8882

neuroreha 2020; 12: 159-162

(c) 2020. Thieme. All rights reserved.

Georg Thieme Verlag KG, Rüdigerstraße 14,

70469 Stuttgart, Germany

ISSN 1611-6496 